\title{
Characteristic Analysis of Wireless Power Transmission System Based on Mutual Inductance Parameters
}

\author{
Dandan Liu ${ }^{\mathrm{a}}$, Xiaodong Zhang ${ }^{\mathrm{b}}$ and Fei Jia \\ Beijing Jiaotong University, Beijing 100044, China \\ a15121435@bjtu.edu.cn, bxdzhang@bjtu.edu.cn, c15121418@ bjtu.edu.cn
}

\begin{abstract}
Keywords: wireless energy transmission, magnetically coupled, mutual inductance, magnetic field
\end{abstract} analysis.

\begin{abstract}
In the paper, we analyze the factors of mutual inductance between two coils in magnetically coupled wireless energy transmission system based on the resonance frequency of 85 $\mathrm{kHz}$. When the radius of transmitter coil remains the same, the simulation analysis of the main geometric parameters such as the radius of receive coil, the axial and radial displacement distance affect the mutual inductance by numerical calculation and finite element simulation. The results show that when the receiving coil radius changing, mutual inductance has a great value, but with the increase of the offset diatance, the mutual inductance decrease rapidly; Through the influence of axial offset and radial offset on mutual inductance and efficiency, the range of dynamic deviation of coil is effectively defined.
\end{abstract}

\section{Introduction}

Magnetic coupling resonant technology is a time-varying electromagnetic field as a medium, using two or more with the same resonant frequency, high quality factor of the electromagnetic resonance system to achieve wireless power transmission technology through the magnetic coupling resonant way [1]. In recent years, the technology has been applied to electric vehicles, medical electronic equipment, portable mobile devices and underwater submarines and other fields [2]. In the wireless power transmission system, the structure and parameter design of the transmitter and the receiver coil are very important to improve the power and efficiency of the wireless power transmission. In the dynamic wireless charging process, the two coils in the axial and radial position will have offset distance, which will affect the coil coupling mutual inductance and then affect the power transmission system [3]. Some scholars have studied the factors of mutual inductance [4] mainly focused on the analysis of mutual inductance of the two coaxial coils. Based on this, the two parallel coils are modeled in the space. Through the numerical calculation and the finite element analysis, the coupling effect of the different coil radius, axial offset and radial offset on mutual inductance are studied, then the influence of mutual inductance on the efficiency of transmission system is analyzed.

\section{Magnetic Coupling Transmission System Model}

\subsection{Equivalent Circuit}

The equivalent circuit of the transmission system is shown in Fig. 1, and the system consists of the transmitting part and the receiving part. The high-frequency power supply is composed of a high-frequency power source and a power amplifier circuit which generate high-frequency time-varying electromagnetic field transmitted to the receiving circuit through the inductive coupling between the coils. In Fig. 1, Rs is the internal resistance of the power supply; $\mathrm{L}_{1}$ is the equivalent inductance of the transmitting coil; $\mathrm{L}_{2}$ is the equivalent inductance of the receiving coil; $\mathrm{R}_{1}$ is the equivalent resistance of the transmitting coil at high frequency; $\mathrm{R}_{2}$ is equivalent resistance of the receiving coil at high frequency; $\mathrm{M}$ is the mutual inductance between the two coils; $\mathrm{C}_{1}$ is the tuning capacitance of the transmitting coil at high frequency; $\mathrm{C}_{2}$ is the tuning capacitance of the receiving coil at high frequency; $\mathrm{R}_{\mathrm{L}}$ is the load resistance. 


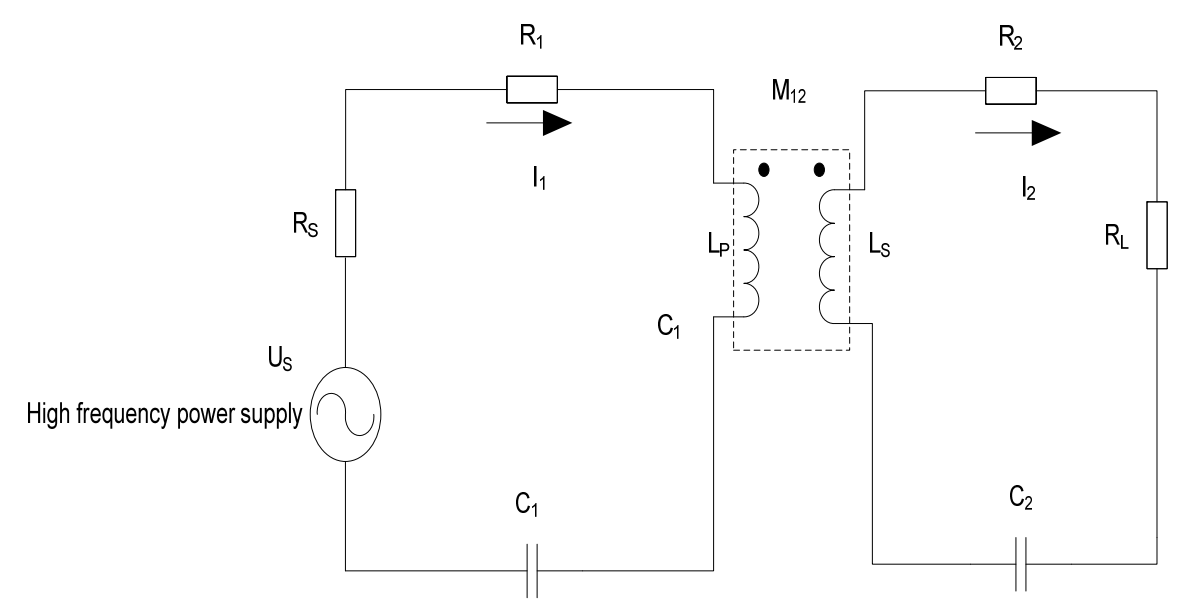

Fig. 1 Equivalent circuit of wireless power transmission system

Figure 1 shows the wireless power transmission system equivalent circuit, calculating the system output power and efficiency [5]:

$$
\begin{aligned}
& P_{\text {in }}=\frac{U_{S}^{2}}{\left|Z_{1}+\frac{(\omega M)^{2}}{Z_{2}}\right|} \\
& P_{L}=I_{2}^{2} R_{L}=\frac{(\omega M)^{2} U_{S}{ }^{2} R_{L}}{\left(Z_{1} Z_{2}+(\omega M)^{2}\right)^{2}} \\
& \eta=\frac{P_{L}}{P_{\text {in }}} \\
& Z_{1}=R_{S}+R_{1}+j \omega L_{1}-\frac{\mathrm{j}}{\omega C_{1}}, Z_{2}=R_{2}+R_{L}+j \omega L_{2}-\frac{\mathrm{j}}{\omega C_{2}} \\
& \mathrm{j} \omega L_{1}-\frac{\mathrm{j}}{\omega C_{1}}=j \omega L_{2}-\frac{\mathrm{j}}{\omega C_{2}}=0 \text {, the expression of efficiency: } \\
& \eta=\frac{R_{L}}{\frac{\left(R_{S}+R_{1}\right)\left(R_{2}+R_{L}\right)^{2}}{(\omega M)^{2}}+R_{2}+R_{L}}
\end{aligned}
$$

The radius of two coils is $r_{1}, r_{2}$ respectively, $h$ is the axial offset of the two coils, $d$ is the radial offset distance, $\theta, \varphi$ are integral factor, the mutual of two coils calculated by the Newman formula [6] is

$M=\frac{u_{0}}{4 \pi} \iint \frac{\mathrm{dl}_{1} \cdot \mathrm{dl}_{2}}{r_{12}}$

$\mathrm{dl}_{1}=r_{1}(-x \sin \theta+y \cos \theta), \quad \mathrm{dl}_{2}=r_{2}(-x \sin \varphi+y \cos \varphi)$

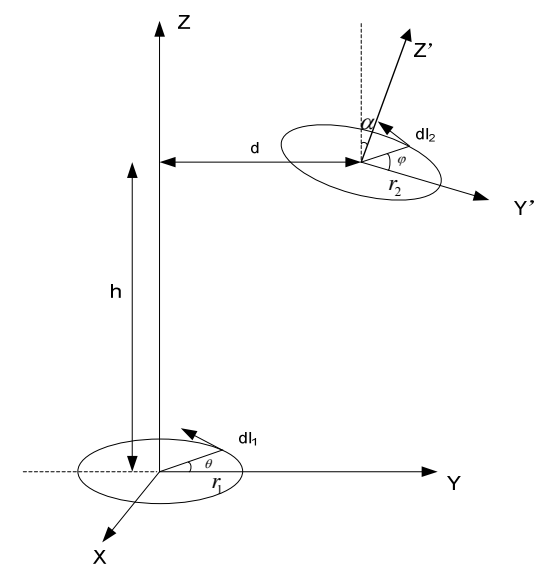

Fig. 2 two any coils in the space 
One point of round 2 in the XYZ coordinates is: $x=r_{2} \cos \varphi, y=r_{2} \sin \varphi, z=0$

When the circle 2 is rotated clockwise along the $x$-axis and the coordinate parallel translated, the coordinates of the point $(\mathrm{x}, \mathrm{y}, \mathrm{z})$ on the circle 2 become $\left(\mathrm{x}\right.$ ', $\left.\mathrm{y}^{\prime}, \mathrm{z}^{\prime}\right)$ :

$$
\begin{aligned}
& \left(\mathrm{x}^{\prime}, \mathrm{y}^{\prime}, \mathrm{z}^{\prime}\right)=(\mathrm{x}, \mathrm{y}, \mathrm{z}) \mathrm{Mx}(-\alpha)+(0, d, h) \\
& x^{\prime}=r_{2} \cos \varphi, y^{\prime}=r_{2} \sin \varphi \cos \alpha+d, \quad \mathrm{z}^{\prime}=-r_{2} \sin \varphi \sin \alpha+h \\
& \mathrm{dl}_{1}=r_{1}(-x \sin \theta+y \cos \theta), \quad \mathrm{dl}_{2}=r_{2}(-x \sin \varphi+y \cos \varphi \cos \alpha-\mathrm{z} \cos \varphi \sin \alpha) \\
& M=\frac{u_{0}}{4 \pi} \int_{0}^{2 \pi} \int_{0}^{2 \pi} \frac{r_{1} r_{2}(\cos \theta \cos \varphi \cos \alpha+\sin \theta \sin \varphi)}{r_{12}} d \theta d \varphi \\
& r_{12}=\sqrt{\left(r_{2} \cos \varphi-r_{1} \cos \theta\right)^{2}+\left(r_{2} \sin \varphi \cos \alpha+d-r_{1} \sin \theta\right)^{2}+\left(-r_{2} \sin \varphi \sin \alpha+h\right)^{2}}
\end{aligned}
$$

when $\alpha=0$, the mutual inductance between the two parallel coils can be obtained

$$
\begin{aligned}
& M=\frac{u_{0}}{4 \pi} \int_{0}^{2 \pi} \int_{0}^{2 \pi} \frac{r_{1} r_{2} \cos (\theta-\varphi)}{r_{12}} d \theta d \varphi \\
& r_{12}=\sqrt{\left(r_{2} \cos \varphi-r_{1} \cos \theta\right)^{2}+\left(r_{2} \sin \varphi+d-r_{1} \sin \theta\right)^{2}+h^{2}}
\end{aligned}
$$

Two single-turn coil mutual inductance is related to coil radius, spacing, coil deflection angle from the mutual of the equation (6).In the process of shifting the coil, the self-inductance and the resonant state of the system does not change, but the mutual inductance changes. From the equation (4) we can see that the change of mutual inductance parameters directly affect the transmission efficiency of the system. Through the analysis and discussion of the factors of mutual inductance, we can analyze the influence of the dynamic change of the coil on the wireless energy transmission efficiency.

\section{The Numerical Calculation of Mutual Inductance}

The direct solution of mutual is difficult by the single turn mutual inductance formula .Using Matlab numerical calculation method can effectively solve the problem of mutual inductance. The spiral coil can be equivalent to a N-turn concentric ring with a current flowing through each loop. First consider the impact of a current-carrying wire, taking the line current Idl. The number of turns on the transmission coil side is $\mathrm{N}_{1}$, and the number of turns on the receiving coil side is $\mathrm{N}_{2}$. The flow chart of the mutual calculation is shown in Fig.3.

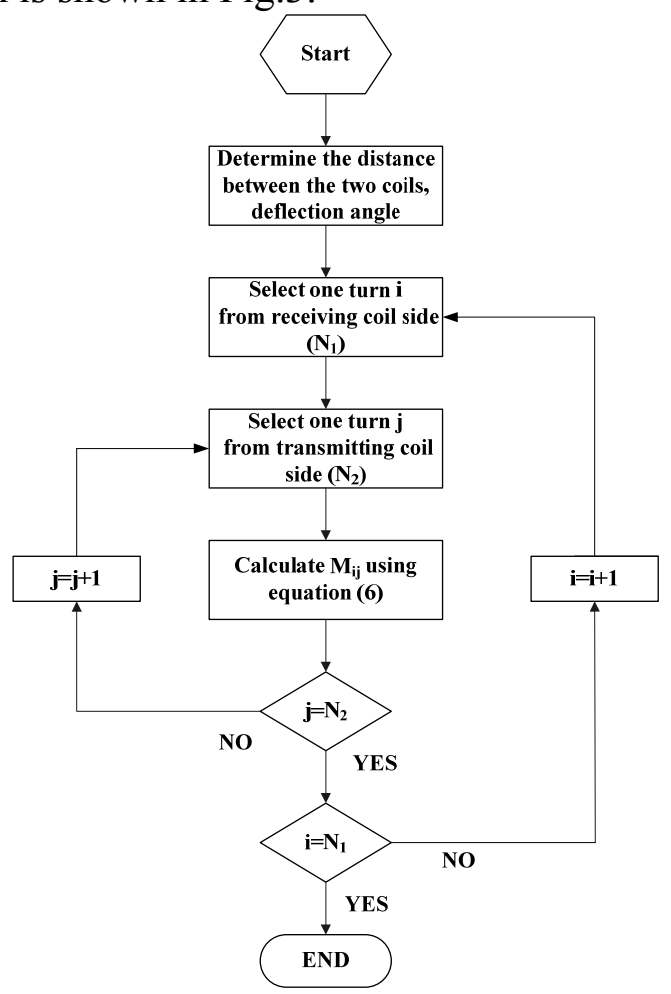

Fig. 3 Two - coil mutual inductance calculation flow chart 
Figure 4 shows two parallel coils, the radius of the transmitting coil $\left(\mathrm{N}_{1}=10\right)$ in the space is fixed, mutual inductance changes with different receiving coil $\left(\mathrm{N}_{2}=10\right)$ radius in axial and radial deviation. The independent variables are axial and radial offset, and the function value is the mutual inductance between the two coils. It can be seen from the change of the curve in the figure that the mutual inductance of the two coils decreases monotonically with the increase of the axial offset. When the radius of the two coils is equal, the mutual inductance is the largest when there is no deviation. But the mutual inductance drops rapidly with the increase of axial and radial offset, which stabilizes after decay to a certain extent. The radius of the two coils is not equal, the mutual inductance between them have a great value when the axial offset is 0 and the radial offset is $\left|r_{1}-r_{2}\right|$.

When improving the mutual inductance between the coils, we need to weigh the coil radius and spacing to determine the best parameters. The radius of the two coils should have little difference, but long-distance transmission will cause a sudden drop in mutual inductance. While the difference between the two coil radiuses is large, the mutual decline gently with the spacing increasing. This provides a theoretical reference for the selection of the wireless transmission coil size and the placement of the spatial location.

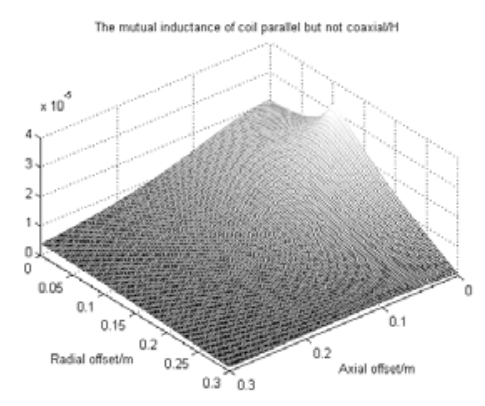

$r_{1}=0.25 \mathrm{~m} \quad r_{2}=0.15 \mathrm{~m}$

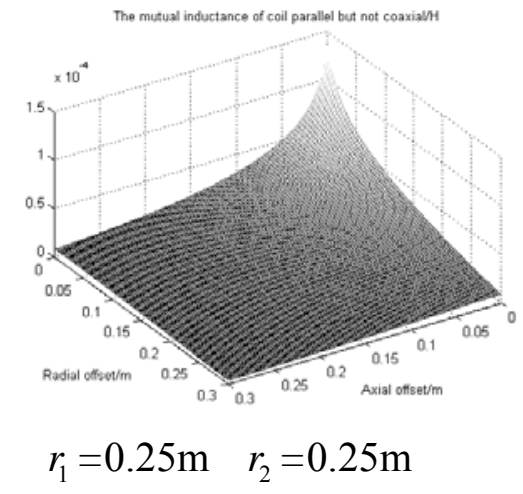

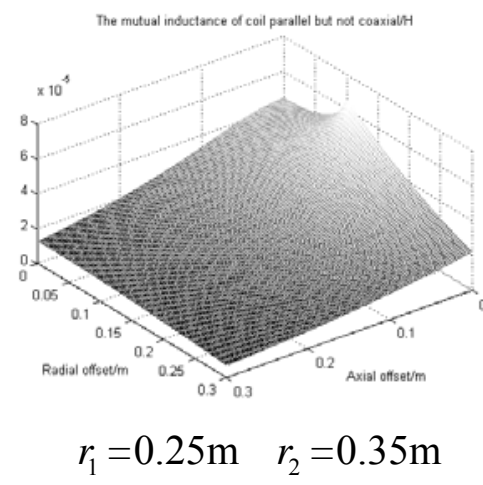

Fig. 4 calculate the mutual inductance value of the two parallel coils

\section{Finite Element Simulation}

Modeling the wireless power transmission system and analyzing the magnetic field around the coil are very complex, which usually including circuit solving and finite element calculation. The solution of the coils includes the following aspects:

a) First, the current flowing in the coils are calculated by a circuit approach;

b) Then, the obtained current are used as the source of the magnetic field for coils in air.

c) Numerical or analytical methods solve the distribution of magnetic field.

\subsection{Magnetic Field Calculation}

The frequency of magnetic coupling resonant is obove the range of $\mathrm{kHz}$, and the wavelength is much larger than the coil size. When the distance from the spot point to the source point is smaller than the wavelength of the field, the field can be regarded as a magnetic quasi-static field, and the presence of the displacement current can be ignored. By the Coulomb specification in [7]:

$\nabla^{2} A=-\mu J$

$J=\gamma E$

The current density $J$ characterizes the current density including $J_{S}$ externally applied and the eddy current density $\mu J_{e}$.

$J_{S}+J_{e}=-\gamma E$

$E=-\nabla \varphi-\frac{\partial A}{\partial t}$

We can get dynamic equation of $A$ in the quasi-harmonic quasi-static field:

$\nabla^{2} A-\mu \gamma \frac{\partial A}{\partial t}=-\mu J_{S}$

Equation (12) can be used to solve the magnetic field distribution around the coils. 


\subsection{Influence of Axial and Radial Offset on the System}

In order to simulate the mutual coupling between the two coils, we can solve the mutual inductance, efficiency of the two coils through the COMSOL finite element analysis software. The current of the transmitter coil is $\mathrm{I}_{1}$, and the receiving coil circuit is set to open. The receiving side of the coil will produce the induced voltage $\mathrm{U}_{2}$, and we calculate the mutual inductance between the two coils in formula $U_{2}=\mathrm{j} \omega M I_{1}$. Based on this, the mutual inductance between the coils is obtained through the excitation parameters of the coil are set and then the dynamic parameters are scanned respectively.

Table 1 Wireless power transmission simulation parameter table

\begin{tabular}{cc}
\hline parameter & size \\
\hline The radius of Transmit coil & $0.25 \mathrm{~m}$ \\
The radius of receiving coil & $0.15 \mathrm{~m}-0.35 \mathrm{~m}$ \\
turns & 10 \\
Coil distance (axial) & $0 \mathrm{~m}-0.3 \mathrm{~m}$ \\
Coil distance (radial) & $0 \mathrm{~m}-0.3 \mathrm{~m}$ \\
frequency & $85 \mathrm{kHz}$ \\
\hline
\end{tabular}

The mutual inductance obtained by finite element calculating the dynamic offset of the coil is consistent with the previous numerical calculation. In order to observe the magnetic field distribution under different receiving coils, Fig. 5 shows the coaxial two coils which the transmitting coil is fixed and the receiving coils are not the same, the magnetic field of which is expressed by natural logarithm. It can be seen from Fig.5 that the receiving coil radius affects the magnetic field distribution. When the radius of the transmitting coil and the receiving coil is the same, the magnetic field distribution between the two is relatively more uniform. The larger the radius of the receiving coil, the larger the effective coupling region of the magnetic field. Similarly, when the receiving coil is small, the effective coupling region is smaller.
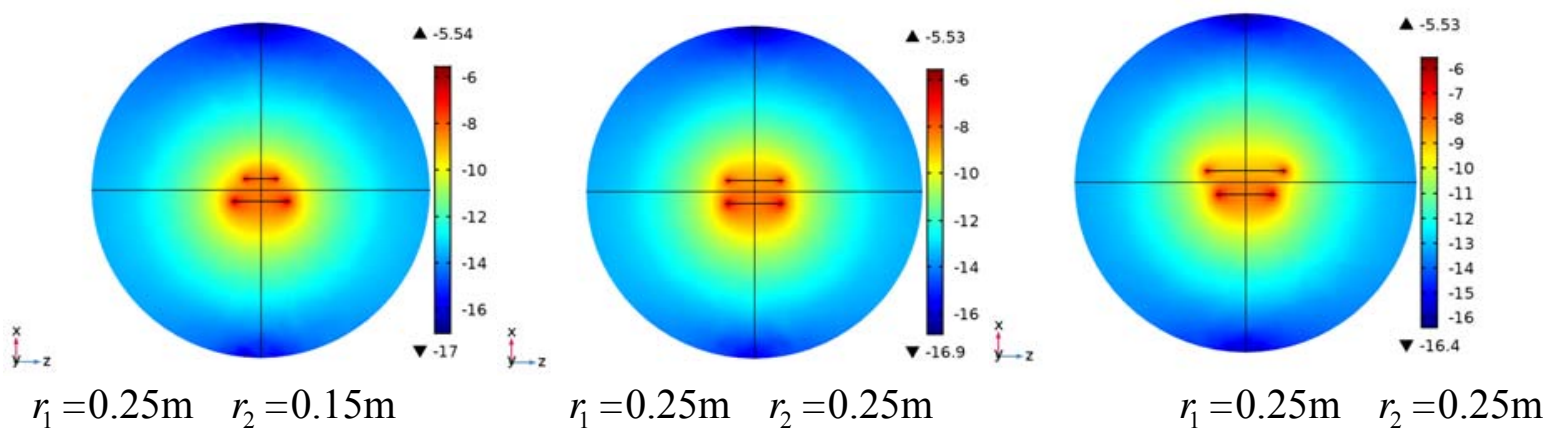

Fig. 5 The distribution of magnetic field under different receiving coils

After the above discussion, the the mutual of two coil radius which the radius is equal obtain a larger value when the transmitter coil and receive coil offset is not big. From equation (4) we can see that the change of efficiency and mutual inductance is consistent. In order to observe the effect of mutual inductance on the efficiency of the system, the radius of the two coils is now set to $r_{1}=r_{2}=0.25 \mathrm{~m}$ and the axial and radial distances between the two coils are set as variables. We calculate system efficiency in the process of dynamic offset by taking (7) into (4) through the parameter scanning method.

When the radius of the transmitting coil and the receiving coil is the same, the mutual between the coils drops with the distance of axial and radial offset increasing. the system efficiency drop with the distance of axial and radial offset increasing in fig.6. When the axial offset is $40 \mathrm{~mm}$ and the radial offset is 0 , the efficiency is $71 \%$. When the radial offset is $r_{1}$, the efficiency is $10 \%$. Therefore, it is beneficial to minimize the axial offset of the two coils and to set the radial deviation within a certain range to increase the coupling inductance between the two coils. 


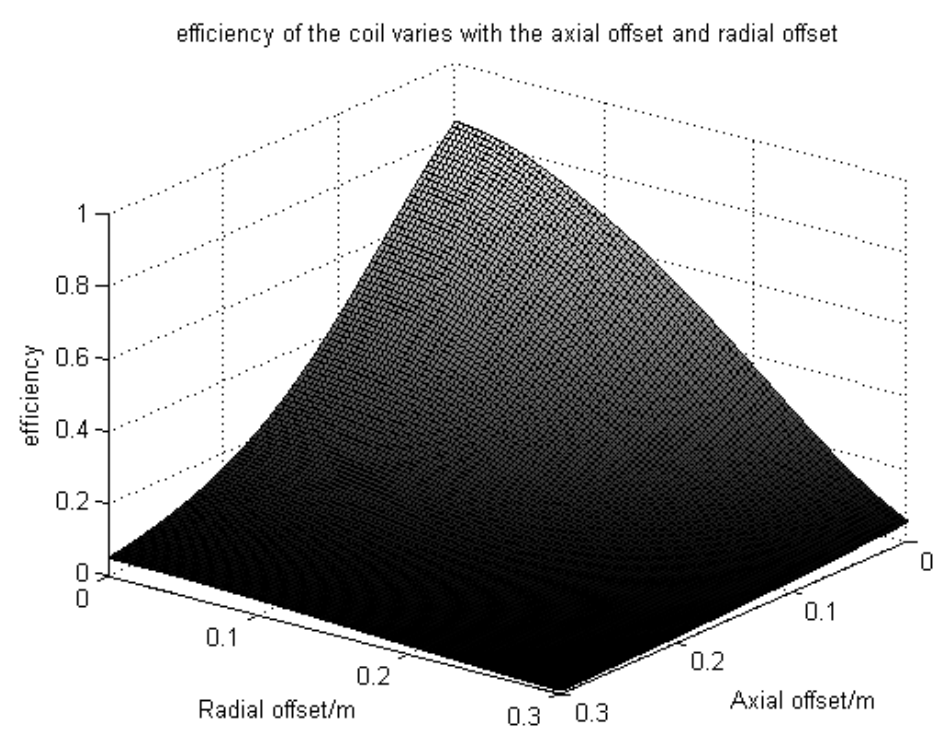

Fig. 6 Efficiency of the coil varies with the axial offset and radial offset

\section{Conclusion}

In this paper, the influence factors of arbitrary two parallel coils in space are analyzed by numerical calculation and finite element simulation. It is found that the radius and offset of the receiving coil are important factors that affect the mutual inductance. We conclude that when the size of the two coils radius is equal, the smaller the offset, the bigger the mutual inductance between the two coils. At the moment, if the distance increasing, mutual inductance drops rapidly. We can optimize the coil structure, the two coils of the same structure can be used to enhance the coupling in the static wireless charging which the coil offset frequency is small. The coil offset frequency increasing in dynamic wireless charging, we can use "big pass small" or "small pass big" coil mode to reduce the attenuation speed of mutual inductance with the distance of displacement.

\section{References}

[1] FAN Xing-ming, MO Xiao-yong, ZHANG Xin, Research status and application of magnetic coupling resonant radio energy transmission, J.Transactions of Electrical Engineering, 28 (2013): 75-82

[2] Zhao Zheng-ming, Liu Fang, CHEN Kai-nan. Study on Research on Wireless Charging Technology of Electric Vehicle, J.Journal of Electrical Engineering, 31 (2016): 30-40

[3] Li Yang, Yang Qing-xin, Yan Zhuo, Analysis of the effective transmission distance of wireless energy and its influencing factors, J. Transactions of Electrical Engineering, 28 (2013): 106-112

[4] XIAO Si-yu, MA Dian-guang, ZHANG Han-hua, Coil optimization of coupled resonant wireless power transmission system , J.Journal of Electrical Engineering, 30 (2015): 221-225

[5] CHEN De-qing, WANG Li-fang, LIAO Cheng-lin, Loss analysis and magnet structure optimization of wireless charging system, J. Journal of Electrical Engineering, 30 (2015) :154-158

[6] Fuxin Liu, Yong Yang, Modeling and optimization of magnetically coupled resonant wireless power transfer system with varying spatial scales, J.IEEE Transactions on Power Electronics, 32(2017): 3240 - 3250

[7] Feng Ci-Zhang,Ma Xi-kui, Introduction to Engineering Electromagnetic Fields, Beijing, 2000 